\title{
DEPENDENCE OF GENOTYPIC VIABILITIES ON CO-EXISTING GENOTYPES IN DROSOPHILA
}

\author{
P. A. PARSONS \\ Deportment of Genetics, University of Combridge
}

Received $17 \cdot x i \cdot 5^{8}$

\section{INTRODUCTION}

Haldane in I924 discussed the concepts of familial selection whereby the size of a family is severely limited by the food supply, more embryos being produced than can exist. In the case of mixed litters, in a mammal for example, there will be some genotypes somewhat weaker or less viable than the others. Haldane gives an illustrative example. He considers three litters of 20 embryos each, the first consisting of wholly strong types, the second of io strong and ro weak, and the third of 20 weak. He then supposes that only io embryos survive and that out of equal numbers, $5^{\circ}$ per cent. more of the strong survive. Thus the survivors are Io strong from the first litter, 6 strong and 4 weak from the second and io weak from the third totalling 16 to I4. If competition had been free, the numbers would have been 18 to i 2 .

In Drosophila pseudo-obscura it has been shown (Birch, 1955) that the main competitive effect is between larvæ, with little competition between adults. Lewontin (1955), using Drosophila melanogaster, made a study of the larval viabilities of twenty-two strains of varying population densities. Larval viabilities varied but usually showed optimal viability at a moderate level of competition. He studied the viabilities of these strains mixed with a white-eyed stock as well, and found a change in the optimal density, some showing increased and some decreased viability compared with the pure strains. The conclusion is that the viability of a genotype is a function of the other genotypes co-existing with it; the result of any particular combination of genotypes not being predictable on the basis of the genotypes tested in isolation.

In a two-point backcross linkage experiment the proportion of the competing genotypes is different in each phase. Considering such an experiment for two factors $a$ and $b$ which are somewhat inviable in the recessive state, four genotypes are obtained in the offspring:

$$
A B / a b, A b / a b, a B / a b, a b / a b \text {. }
$$

In coupling, the parentals are $A B / a b$ and $a b / a b$, the most and least viable genotypes respectively, and if competition is severe, there would be a large elimination of $a b / a b$. In the repulsion phase, the parentals are $A b / a b$ and $a B / a b$, and assuming approximately equal inviabilities of $a$ and $b$ there will be no great difference in elimination between these. The recombinants $A B / a b$ and $a b / a b$, which are fewer in number will 
not be in competition with each other to any great extent but rather with the parentals and as a result the proportion of $a b / a b$ offspring eliminated will not be as grcat as in the coupling phase. Hencc in such a situation, the ratio of $A B / a b: a b / a b$ individuals will vary according to whether they are parentals or recombinants. The ratio of $A b / a b: a B / a b$ should not vary much as in both phases they will be eliminated approximately equally. However, if one of the recessive factors is very much morc inviable than the other, both ratios may be expccted to vary between coupling and repulsion. If only one factor is of poor viability, the other being entirely equivalent to the normal allcle under severc competition, there would be no effect, as the viability of this factor would be cqually distributed in each complementary pair. Under normal laboratory conditions of low competition these viability effects would in most situations be cxpected to be negligible.

Two series of two-point backcross cxpcriments were therefore sct up in Drosophila for three levels of competition to study the effect of competition on genotypic viabilities in coupling and repulsion. A preliminary report of one of the expcriments has been published (Parsons, I $\left.95^{8}\right)$.

\section{METHOD}

The two pairs of linked factors selected wcre white $(w)$ and miniaturc $(m)$ on the X-chromosome, and black $(b)$ and vestigial $(\mathrm{vg})$ on chromosome II. Both $w$ and $m$ under optimal conditions of abundant food show little viability effect. Vestigial, however, is somcwhat inviable under the best of conditions and black is probably equivalent to wild-type. Thus the two pairs of factors selected exhibit different viability relationships and should lead to somewhat different results.

Flies were aged for 72 hours prior to mating to eliminate major age differcnces and then mated for 48 hours. The temperaturc was standardised at $25 \pm \mathrm{I}^{\circ} \mathrm{C}$. The three levels of competition were :

(I) One pair of flies matcd in the normal half pint milk bottles with excess food (6o-7o gm.). The medium used was composed of semolina, treacle and agar and was seeded with a few drops of yeast suspension.

(2) One pair of flics in small vials $\left(7 \frac{1}{2} \times 2 \frac{1}{2} \mathrm{cms}\right.$.) with $5 \frac{1}{2} \mathrm{gms}$. of food.

(3) Six pairs of flics in small vials $\left(7 \frac{1}{2} \times 2 \frac{1}{2} \mathrm{cms}\right.$.) with $5 \frac{1}{2} \mathrm{gms}$. of food.

For simplicity these three levels of competition will be termed low, medium, and high in the remainder of the paper.

The offspring from all matings were counted daily as they emerged.

\section{DATA FOR WHITE (w)-MINIATURE (m)}

The data for the three levels of competition arc summarised in table I. Assuming no competition between larvæ, the ratios wm : ++ and $w+:+m$ should not vary between coupling and repulsion. A simple contingency $\chi^{2}{ }_{1}$ may therefore be donc within each lcvel to test this and these tests are presented in table 2.

The only significant result is for the high lcvel of competition. In the low and medium levels genotypic viabilities do not vary appreciably between coupling and repulsion. Examination of table I shows that 
$w m$ flies are of much poorer viability than ++ at the high level in coupling, but in repulsion wm flies are, if anything, more viable than ++ flies. The ratio of $w+:+m$ flies is, however, approximately the same in coupling and repulsion.

TABLE I

Data for w-m

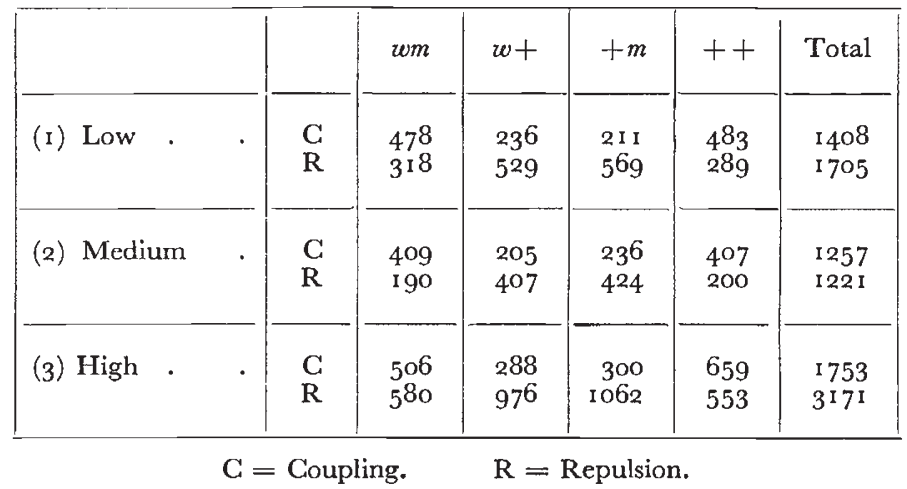

The significance of the $w m:++$ contrast may be interpreted as being due to differential competition between larvæ according to the proportions of the competing genotypes. In coupling the main competition is between wm and ++ so that wm flies are eliminated. In repulsion the general level of competition is lower as it is determined by the $w+$ to $+m$ contrast and thus the rate of elimination of $w m$ compared with ++ is lower than in coupling. This is the type of litter competition postulated by Haldane (I924) and discussed in the introduction.

TABLE 2

$\chi^{2}{ }_{1}$ tests for $\mathrm{wm}:++$ and $\mathrm{w}+:+\mathrm{m}$ within each level of competition

\begin{tabular}{|c|c|c|c|c|c|c|}
\hline & & & \multicolumn{2}{|c|}{$w m:++$} & \multicolumn{2}{|c|}{$w+:+m$} \\
\hline Low & - & . & $\begin{array}{r}\chi^{2}{ }_{1} \\
0 \cdot 71\end{array}$ & $\begin{array}{c}P \\
0.3-0.5\end{array}$ & $\begin{array}{r}\chi^{2}{ }_{1} \\
2 \cdot 53\end{array}$ & $\underset{0 \cdot I-0.2}{P}$ \\
\hline Medium & . & . & $0 \cdot 16$ & $0.5-0.7$ & 0.62 & $0.3-0.5$ \\
\hline High & • & • & $13.5^{6}$ & $<0.00 \mathrm{I}$ & 0.18 & $0.5-0.7$ \\
\hline
\end{tabular}

It is of some interest to find out what effect, if any, competition between genotypes has on the recombination value. In table 3 , estimates of recombination value are given for the coupling and repulsion data with a combined estimate using the product formula (Fisher, I935-53) for each level of competition. Examination of this table shows that the combined estimates are all close to each other but between coupling and repulsion there is some variation for each level 
of competition. Hence, if balanced data are collected the rccombination fraction ought to be reasonably accurate. Bodmer (1959) in some artificially constructed examples has shown that this is so.

TABLE 3

Recombination values between $\mathrm{w}-\mathrm{m}$

\begin{tabular}{|c|c|c|c|c|c|}
\hline & & & Coupling & Repulsion & Combined estimate \\
\hline Low & . & . & $31 \cdot 75 \pm 1 \cdot 24$ & $35 \cdot 60 \pm 1 \cdot 16$ & $33.65 \pm 0.85$ \\
\hline Medium & . & . & $35 \cdot 08+1 \cdot 35$ & $3 I \cdot 94+: I \cdot 33$ & $33 \cdot 49+0 \cdot 95$ \\
\hline High & . & . & $33 \cdot 54 \pm 1 \cdot 13$ & $35 \cdot 73 \pm 0 \cdot 85$ & $34.63 \pm 0.71$ \\
\hline
\end{tabular}

Finally, the analysis of $\chi^{2}$ of these data as proposed by Bodmer and Parsons (1959) for balanced three-point linkage tests is given in tablc 4 . For the analysis, the data are arranged in $2 \times 2$ Latin squarcs $A B$, where $A$ represents onc complementary pair say $w m$ and ++ and $B$ the other.

TABLE 4

$\chi^{2}$ analysis of $\mathrm{w}-\mathrm{m}$ data

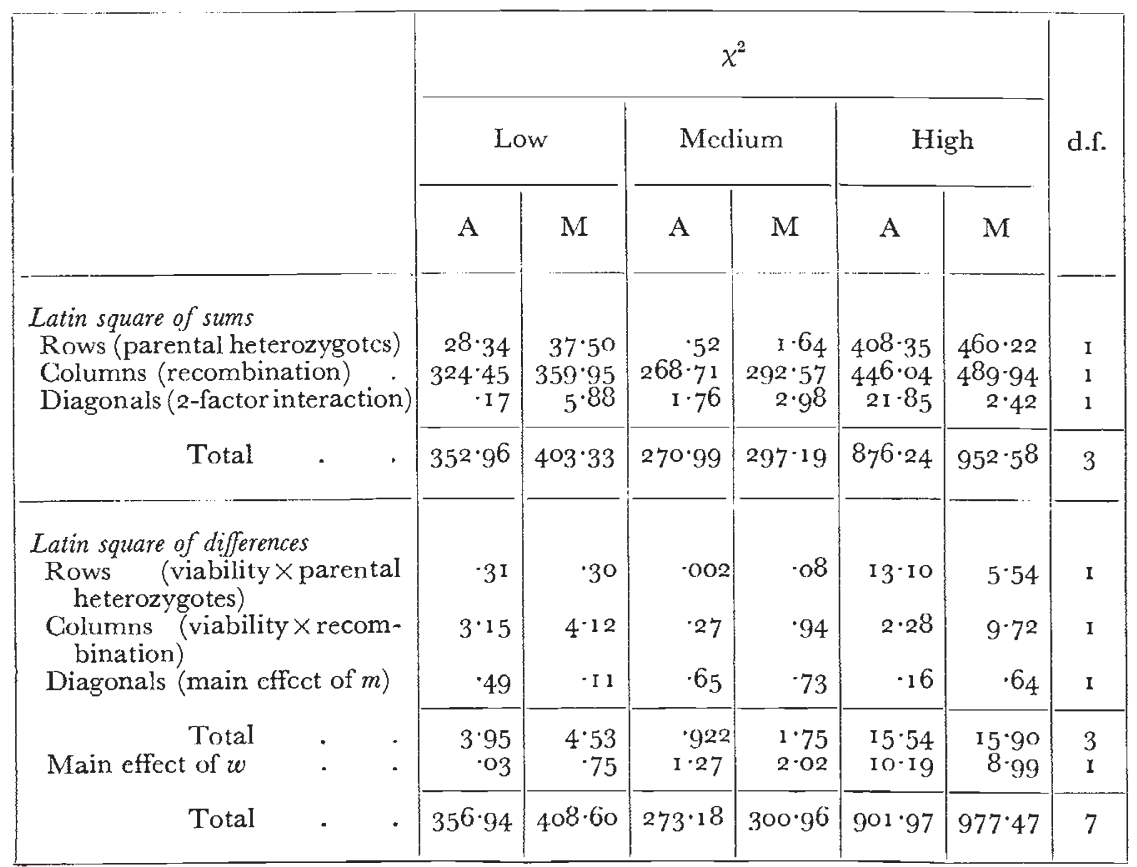

$\mathrm{A}=$ Additive $; \mathrm{M}=$ Multiplicative (analysis after taking logarithms).

There is a total of 7 degrees of freedom as there are 8 observed totals in a $2 \times 2$ Latin square split into complementary genotypes. The 7 degrees of frecdom may be split into 3 for a Latin square of sums 
(formed from the totals for each complementary pair), 3 for a Latin square of differences (formed from the differences between members of a complementary pair), and the remaining degree of freedom is for the sum of squares for the total of the Latin square of differences. All the major sources of variation can be isolated using this analysis provided that there are no major deviations from orthogonality (i.e. equality of the row totals of the Latin square of sums) when a weighted analysis would probably be somewhat more accurate.

The viability $\times$ parental heterozygote interaction may be interpreted as a component to detect whether viabilities vary between coupling and repulsion, and is in fact a component measuring differential competition according to the proportion of competing genotypes present. The viability $\times$ recombination interaction detects whether recombinants or non-recombinants have different viabilities and it is difficult to conceive a biological meaning for it. However, both of these components must be considered together, for, according to whether one gene is more or less viable than the other, these two interactions become interchanged due to the symmetry of the problem. Hence significance of either or both interactions is in itself evidence for a competition effect.

As in the three-point situation, the S.S. may be converted into $\chi^{2}$ values by dividing by the mean of the observations. Such $\chi^{2}$ values are tabulated in table 4 .

At the low level of competition by far the largest $\chi^{2}{ }_{1}$ is for recombination (table 4 ). The only other $\chi^{2}{ }_{1}$ of any magnitude is that for parental heterozygotes which merely reflects the inequality of the row totals. For the medium level, with the exception of the recombination $\chi^{2}{ }_{1}$, the other $\chi^{2}{ }_{1}$ 's are not significant.

However, for the high level, with the exception of the main effect of $m$ and the viability $\times$ recombination $\chi^{2}{ }_{1}$, the remainder of the $\chi^{2}{ }_{1}$ 's are large. The $\chi^{2}{ }_{1}$ for recombination and parental heterozygotes are, as expected, very large. Then at a lower level of magnitude come the $\chi^{2}{ }_{1}$ 's for the two-factor interaction, main effect of $w$, and viability $\times$ parental heterozygote interaction. This analysis shows the predicted sources of variation and as expected demonstrates the validity of the larval competition effect.

The analyses of $\chi^{2}$ therefore confirm that there is a competition effect at the high level but not at the medium or low levels. Furthermore, there is a wm interaction at the high level which is not present at the two lower levels.

Table 4 also gives the analysis of $\chi^{2}$ after taking logarithms which assumes that viabilities act multiplicatively. It is possible that the multiplicative model may represent the mode of action of the viabilities more correctly than the additive model (Bodmer, 1959). At the high level both the viability $\times$ heterozygote and viability $\times$ recombination interactions are significant so that the competition effect is still present. The wm interaction, is, however, reduced to insignificance. At the 
low level, a significant $w m$ interaction and viability $\times$ recombination intcraction $(P<.05)$ becomc apparent. The latter, although not nearly as great as for the high level does indicatc a slight difference betwecn coupling and repulsion. It is, of course, difficult to say whether the additive or multiplicative model is more correct, although the latter seems intuitively more correct, and so a borderline case as this must not be regarded as real evidence.

\section{DATA FOR BLACK (b)-VESTIGIAL ( $v g$ )}

The data for the three lcvels of competition are summarised in table 5. On the whole the data are smaller than for $w-m$ due to poor vg viability.

TABLE 5

Data for b-vg

\begin{tabular}{|c|c|c|c|c|c|c|}
\hline & & $b v g$ & $+v g$ & $b+$ & ++ & Total \\
\hline Low & $\begin{array}{l}\mathrm{C} \\
\mathrm{R}\end{array}$ & $\begin{array}{r}3.54 \\
43\end{array}$ & $\begin{array}{r}4^{8} \\
3^{22}\end{array}$ & $\begin{array}{r}52 \\
39^{2}\end{array}$ & $\begin{array}{c}37^{8} \\
59\end{array}$ & $\begin{array}{l}832 \\
816\end{array}$ \\
\hline Medium & $\begin{array}{l}\mathrm{C} \\
\mathrm{R}\end{array}$ & $\begin{array}{r}314 \\
49\end{array}$ & $\begin{array}{r}45 \\
329\end{array}$ & $\begin{array}{r}49 \\
322\end{array}$ & $\begin{array}{r}402 \\
4^{8}\end{array}$ & $\begin{array}{l}810 \\
74^{8}\end{array}$ \\
\hline High & $\begin{array}{l}\mathrm{C} \\
\mathrm{R}\end{array}$ & $\begin{array}{l}490 \\
143\end{array}$ & $\begin{array}{r}69 \\
1,127\end{array}$ & $\begin{array}{r}102 \\
1,200\end{array}$ & $\begin{array}{r}836 \\
145\end{array}$ & $\begin{array}{l}1,497 \\
2,615\end{array}$ \\
\hline
\end{tabular}

$\mathrm{C}=$ Coupling; $\mathrm{R}=$ Repulsion.

In table 6 , the contingency $\chi^{2}{ }_{1}$ 's for the two comparisons bvg : ++ and $+v g: b+$ are given. The high level shows significance for both comparisons but the low and medium levcls do not show significance.

TABLE 6

$\chi^{2}{ }_{1}$ tests for bvg $:++$ and $+\mathrm{vg}: \mathrm{b}+$ within each level of competition

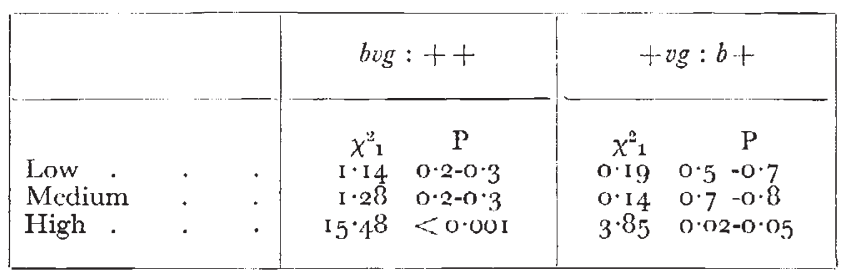

For the high level in coupling, the viability contrast $+v g: b+$ is almost as severc as $b v g:++$, thus showing the poor viability of $v g$ compared with the almost normal $b$. In repulsion, however, wherc the $+v g: b+$ contrast is dominant, $b v g$ and ++ are almost equal and there is a slight deficiency of $+v g$ compared with $b+$. The two sets of data thus exhibit cntircly different genotypic viabilities. In 
general, the situation as presented here, where one factor is of poor viability and the other of only slight inviability might well lead to significant differences between both contrasts in coupling and repulsion, which is in distinction to the wm data where $w$ and $m$ inviabilities were more equal and only the extreme wm : ++ contrast varied.

TABLE 7

Recombination values between $\mathrm{b}-\mathrm{vg}$

\begin{tabular}{|c|c|c|c|c|}
\hline & & Coupling & Repulsion & Combined estimate \\
\hline Low & • & I $2 \cdot 02 \pm I \cdot 13$ & $12 \cdot 5^{\circ} \pm \mathrm{I} \cdot \mathrm{I} 6$ & I $2 \cdot 26 \pm 0.8$ I \\
\hline Medium & . & I I $60 \pm I \cdot I 3$ & I $2 \cdot 97 \pm \mathrm{I} \cdot 23$ & $12.27 \pm 0.83$ \\
\hline High & . & I I. 42 土0. 82 & I I $0 \mathrm{I} \pm 0.6 \mathrm{I}$ & I I. $22 \pm 0.5$ I \\
\hline
\end{tabular}

TABLE 8

$\chi^{2}$ analysis of $\mathrm{b}-\mathrm{vg}$ data

\begin{tabular}{|c|c|c|c|c|c|c|c|}
\hline & \multicolumn{6}{|c|}{$\chi^{2}$} & \multirow{3}{*}{ d.f. } \\
\hline & \multicolumn{2}{|c|}{ Low } & \multicolumn{2}{|c|}{ Medium } & \multicolumn{2}{|c|}{ High } & \\
\hline & A & M & A & M & A & $\mathbf{M}$ & \\
\hline $\begin{array}{l}\text { Latin square of sums } \\
\text { Rows (parental hetero- } \\
\text { zygotes) }\end{array}$ & & $\cdot 04$ & & 30 & & $33^{2} \cdot 4^{8}$ & I \\
\hline Columns (recombination) & $939^{\circ} 04$ & $1602 \cdot 5 \mathrm{I}$ & $887 \cdot 66$ & $\mathrm{I} 5 \mathrm{O} \cdot \mathrm{O} \cdot \mathrm{O} 2$ & $24^{80} \cdot 94$ & $43^{6} 4 \cdot 26$ & I \\
\hline $\begin{array}{l}\text { Diagonals (2-factor inter- } \\
\text { action) }\end{array}$ & $\cdot 24$ & $\cdot 14$ & 2.97 & I. 40 & 190.04 & 83 & I \\
\hline Total & $939 \cdot 44$ & $1602 \cdot 69$ & $893 \cdot 10$ & $1503 \cdot 72$ & $2974 \cdot 95$ & $4697 \cdot 57$ & 3 \\
\hline $\begin{array}{l}\text { Latin square of differences } \\
\text { Rows (viability } \times \text { paren- }\end{array}$ & $2 \cdot 04$ & $3 \cdot 4^{8}$ & $6 \cdot 4^{2}$ & $3 \cdot 4^{I}$ & $22 \cdot 47$ & $46 \cdot 25$ & I \\
\hline $\begin{array}{l}\text { tal heterozygotes) } \\
\text { Columns (viability } \times \\
\text { recombination) }\end{array}$ & $3 \cdot 32$ & $\cdot 46$ & 3.91 & $\cdot 63$ & $35 \cdot 86$ & $2 \cdot 3^{8}$ & I \\
\hline $\begin{array}{l}\text { Diagonals (main effect } \\
\text { of } b \text { ) }\end{array}$ & $\cdot 70$ & $\cdot 29$ & $5 \cdot 20$ & $\cdot 65$ & I $4 \cdot 24$ & $\cdot 57$ & I \\
\hline $\begin{array}{c}\text { Total } \\
\text { Main effect of } v g \text {. }\end{array}$ & $\begin{array}{l}6 \cdot 06 \\
7 \cdot 89\end{array}$ & $\begin{array}{r}4 \cdot 23 \\
11 \cdot 17\end{array}$ & $\begin{array}{r}15.53 \\
4.53\end{array}$ & $\begin{array}{l}4 \cdot 69 \\
2 \cdot 05\end{array}$ & $\begin{array}{l}72 \cdot 57 \\
50^{\circ} \cdot 13\end{array}$ & $\begin{array}{l}49 \cdot 20 \\
64 \cdot 47\end{array}$ & $\begin{array}{l}3 \\
\mathrm{I}\end{array}$ \\
\hline Total & $953 \cdot 39$ & I618.09 & 913.16 & $1510 * 45$ & $3097 \cdot 65$ & $4^{8 I I} \cdot 24$ & 7 \\
\hline
\end{tabular}

$\mathrm{A}=$ Additive $; \mathrm{M}=$ Multiplicative (analysis after taking logarithms).

As in the wm experiment, the estimation of recombination is not affected significantly by competition (table 7). If $v g$ were the sole cause of viability effects, which is probably true at the two lower levels of competition, then such an effect would be distributed 
cqually in each pair of complementary genotypes and the resultant estimatc of recombination would be completely unbiased.

In table 8 , the analyscs of $\chi^{2}$ of these data are given assuming both the additive and multiplicative models. Considering the additive model first, all levels give a significant $\chi^{2}{ }_{1}$ for recombination and main effect of $v g$. The medium level gives, as well as these, significant $\chi^{2}{ }_{1}$ 's for the viability $\times$ hetcrozygote and viability $\times$ recombination interactions, and the main effect of black. The high level givcs significance for all components. Hence a compctition effect is indicated for the medium and high levcls.

The multiplicative model provides an intercsting contrast. At all levels the recombination effect rcmains and at the low level the $v g$ effect. At the high level a very large $b$-vg interaction and viability $x$ rccombination interaction become insignificant. The viability $\times$ hetcrozygote intcraction, main effect of $v g$, recombination, and parental hetcrozygote effect remain. At thc medium level the only significant $\chi^{2}{ }_{1}$ is for recombination. Throughout the thrce levels the change of modcl has the effect of reducing the viability $\times$ recombination $\chi^{2}{ }_{1}$.

Hence both models give a significant compctition effect at the high level but the logarithmic analysis does not give a competition effect at the medium level. The change of scale therefore alters the magnitude of some interactions but does not remove the scvere competition effcct found at the high level.

For the high level, the $b$-vg interaction is not significant on the multiplicative model, but is highly significant on the additive model. This shows that for these data, the multiplicative model is probably more correct than the additive model.

\section{DISCUSSION}

These data show that genotypic viabilities vary according to the proportions of the compcting genotypes if competition is severe cnough.

Of the two linkage tests presented, the date for the two factors $w$ and $m$ arc somewhat more conclusive. 'They are separated by a recombination fraction between 30 per cent. and 35 per cent. so ensuring large numbers in the recombinant classes such that a genotypic viability effect can be found more easily. It secms that this recombination fraction may be optimal for detecting genotypic viability effects. For recombination fractions of 50 per cent., or indepcndence, all four genotypes would be present in equal proportions so eliminating any differential effect between coupling and repulsion. In the casc of small rccombination fractions, large numbers need to be bred to get adequate totals in the recombinant classes.

It would be of interest to carry out further two-point or even three-point tests varying the levels of competition. A quick survey of the published linkage data in Drosophila did not reveal any suggestive 
evidence of differential genotypic viabilities, but this is probably because linkage studies are not as a rule done under very crowded conditions.

Of secondary interest is the effect of changing the model by which the genes act from an additive to a multiplicative model. This change alters the magnitude of the gene effects and the various interactions but the main conclusions concerning competition hold irrespective of scale. However, in borderline cases, a change in model could result in different interpretations. Biologically, it seems more correct to say that two genes act multiplicatively. For example, if two genes are of viability 0.5 , then on the additive scheme the double recessive would be lethal, and on the multiplicative scheme its viability would be 0.25 . In the limit, therefore, the multiplicative scheme is favoured. Each case must, however, be judged on its own merits as genes react differently according to environmental factors and the genetic background.

The implications of viabilities of genotypes varying according to the competing genotypes present has not, as yet, been stressed much. In natural conditions, where competition is much more severe than in the artificial and optimal conditions created in the laboratory such effects must assume some importance. Selective advantages and disadvantages of a gene or genotype must be considered in relation to the food available and the types and proportions of the other genotypes present in the population. This is necessarily complex, but only by making the " model" complex can a true picture of the actual system be obtained.

\section{SUMMARY}

Two-point backcross experiments were done in coupling and repulsion for two pairs of factors in $D$. melanogaster at three levels of competition between larvæ.

One experiment was for white $(w)$ and miniature $(m)$. At the highest level of competition, the ratio of the extreme types wm and ++ differed in coupling and repulsion, but the ratio $w+:+m$ was the same in both phases.

The second experiment was for black $(b)$ and vestigial $(v g)$. At the highest level of competition, the ratios of both bvg: ++ and $b+:+v g$ varied according to the phase of the experiment. The difference between this and the wm experiment is because $v g$ is rather inviable and $b$ almost normal, which is a more unbalanced arrangement of viabilities than for wm.

The variable viability according to the phase of the experiment is shown to be due to competition with other genotypes, the proportion of the genotypes differing in coupling and repulsion. Thus the viability of a genotype is dependent on the proportion of the other genotypes co-existing with it. 
The data are analyscd by application of $\chi^{2}$ technique to the original data and after taking logarithms; thus changing from an additive to a multiplicative model. It is probable that the multiplicative model is more correct as some of the interactions are smaller than on the additive model.

Acknoweledgment.--I wish to thank $\mathrm{Mr} \mathrm{S}$. A. Mallyon for his very competent assistance throughout the course of this work.

\section{REFERENCES}

BIRCH, L. C. I955. Selection in Drosophila pseudo-obscura in relation to crowding. Evolution, 9, 389-399.

BODMER, W. F. I959. Multiplicative effects and the logarithmic transformation in the analysis of balanced multi-point linkage tests. Heredity, I3 $_{3}$ I 57-164.

BODMER, W. F., AND PARSONS, P. A. I959. The analogy between factorial experimentation and balanced multi-point linkage tests. Heredity, ${ }_{13}, 145^{-1} 5^{6}$.

FISHER, R. A. I935-53. The Design of Experiments. Edinburgh : Oliver and Boyd.

HALDANE, J. B. S. I924. A mathematical theory of natural and artificial selection. Trans. Cambridge Phil. Soc., 23, 19-41.

LEWONTIN, R. C. 1955. The effccts of population density and competition on viability in Drosophila melanogaster. Evolution, 9, 27-41.

PARSONS, P. A. I958. Competition between genotypes in Drosophila melanogaster. Nature, $182,27 \mathrm{I}$. 